\title{
As Contribuições do Estudo Extra Classe nas Notas Escolares dos Alunos de uma Escola da Cidade de Barra do Bugres - Mato Grosso
}

The Contributions of the Extraclass Study in the Notes of Students of a School at the Barra do Bugres - Mato Grosso

Welvesley Silva Santos, Edinéia Aparecida dos Santos Galvanin e Jaqueline Nunes Carvalho

Universidade do Estado do Mato Grosso (UNEMAT), Brasil

welvesleysantos@gmail.com; galvanin@gmail.com; jaquelinencarv@gmail.com

\section{Resumo}

Pesquisas apontam que existem no Brasil mais de 16 milhões de pessoas analfabetas, e o Programa Internacional de Avaliação de Alunos identificou que a qualidade da Educação brasileira ainda é um grande problema. Assim, essa pesquisa tem por objetivo analisar o tempo de estudo extraclasse como fator de sucesso no processo de aprendizagem na disciplina de Matemática dos alunos do segundo ano de uma Escola Estadual da cidade de Barra do Bugres, Mato Grosso. Para alcançar nosso objetivo, optamos pela metodologia quantitativa. Para tal, utilizamos para análise dos dados a correlação de Spearman, regressão linear, o teste F e o teste $t$ de Student. Os resultados indicaram que o estudo extraclasse e a utilização da internet em relação aos conteúdos de Matemática têm contribuído para o desempenho dos alunos na disciplina em questão.

Palavras-chave: Estudo extraclasse; Desempenho escolar; Matemática; Métodos estatísticos

\section{Abstract}

Researches show that there are in the Brazil more than 16 million people illiterate and the International Student Assessment shows that the level of quality in the Brazilian Education is still a big problem. Thus, this research has the objective to analyze the time of extraclass study as a success factor in the learning process in the Math discipline of the students of second year of a State School in the city of Barra do Bugres, Mato Grosso. To achieve our goal, we opted for the quantitative methodology, we use the Spearman correlation, linear regression, the F-test and Student's $t$-test for the data analysis. After the analysis we concluded that the extraclass study in relation to the contents of Math has contributed to the performance of students in the discipline referred to, and the use of the internet with the same purpose has collaborated for the performance of students in the same subject.

Keywords: Extraclass study; School performance; Math; Statistical methods 


\section{Introdução}

Muitas pesquisas sobre a qualidade da Educação no Brasil estão sendo realizadas, como por exemplo, o resultado do Programa Internacional de Avaliação de Alunos (PISA) (BRASIL, 2012), no qual o Brasil obteve as seguintes posições: $58^{\mathrm{a}}$

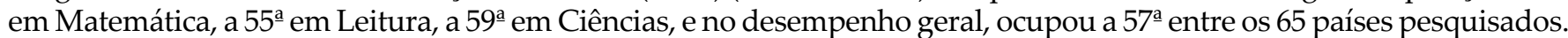

Nesse contexto, a baixa qualidade da Educação no Brasil é apontada como empecilho na solução de problemas econômicos e sociais, tais como a desigualdade da distribuição de renda, os altos índices de pobreza, entre outros.

Segundo (GONÇALVES et al., 2016, p. 02) "vários aspectos são considerados influentes no grau de rendimento do aluno, tais como: participação da família, professor/instituição de ensino, autoestima do aluno, saúde geral do aluno, tempo dedicado ao estudo dentro e fora de sala de aula, entre outros fatores".

Consideramos ser relevante o tempo de dedicação do aluno fora da sala de aula em estudos extraclasse, com o intuito de complementar e aprofundar os conhecimentos sobre assuntos expostos preliminarmente pelos professores. Dessa forma, o educando pode ter influência desse tipo de estudo na construção do saber, e pode até mesmo obter resultados expressivos nas avaliações formais realizadas com o intuito de investigar o nível de assimilação/compreensão dos novos conteúdos apresentados (GONÇALVES et al., 2016).

Nessa perspectiva, uma das tentativas para melhorar a qualidade do ensino e aprendizagem é a utilização das Tecnologias Digitais da Informação e Comunicação (TDIC) pelos alunos e professores nas escolas, tanto nos momentos em sala de aula como nos estudos extraclasse. O uso das TDIC como suporte às aulas pode fomentar um ensino e o aprendizado mais dinâmico e atrativo, possibilitando uma interação maior entre os envolvidos no processo, tornando assim, a escola mais agradável para os alunos (ALMEIDA, 2013).

A internet é uma das ferramentas das TDIC que pode auxiliar os professores nesse trabalho, pois assume um papel de motivadora de alterações, por ser uma rede inacabada e em constante evolução. Deve-se à internet o número elevado de utilizadores das tecnologias digitais, o aumento da sociedade da informação e comunicação, e as transformações nos espaços educativos. Esta, por sua vez, possibilita que o aluno acesse as informações que quiser e a qualquer momento, o que possibilita que o educando se torne mais autônomo no processo de ensino-aprendizagem. Assim, a construção do conhecimento não fica atrelada somente à fala do professor, tampouco à sala de aula (POCINHO, 2012).

As TDIC, precisamente a internet, proporciona aos estudantes a oportunidade de acesso aos sites de pesquisa, e a participação em redes sociais e fóruns de discussões das diversas áreas de conhecimento. Assim, o estudante deixa de ser um mero espectador e passa a construir o seu próprio conhecimento. O professor deixa de ser um transmissor de conhecimento e passa a motivar, incentivar e auxiliar os alunos não só no uso das tecnologias, mas também, nas técnicas de pesquisa.

Nesse contexto, o papel do professor é muito importante, pois muitos alunos não têm conhecimento de como, onde e por que pesquisar. Então, cabe ao professor exercer os papeis de orientador, incentivador e auxiliador, funções essas que são de extrema importância para a construção do conhecimento dos alunos, motivando-os, então, a utilizar a internet, também, nos momentos de estudos extraclasse.

Concernente a isso, esse trabalho tem como objetivo analisar o tempo de estudo extraclasse como fator de sucesso no processo de aprendizagem na disciplina de Matemática dos alunos do segundo ano de uma Escola Estadual da cidade de Barra do Bugres, Mato Grosso.

\section{Materiais e Método}

Considerando nosso objetivo, optamos em utilizar a abordagem quantitativa. Essa utiliza-se da quantificação de dados numéricos e embasa-se em métodos estatísticos. Sendo assim, na pesquisa realizada, utilizamos uma abordagem probabilística com a população dos alunos do $2^{\circ}$ ano do Ensino Médio da escola pesquisada.

Com os avanços das tecnologias digitais os pesquisadores utilizam softwares para o bom desempenho das pesquisas, e juntamente com esses avanços, os softwares específicos para métodos estatísticos têm aumentado significativamente, sendo possível dessa forma, utilizar os métodos de análise descritiva e multivariada, entre outros.

Em nossa pesquisa utilizamos o software Minitab 16 e o Excel, por serem de fácil manipulação e por fornecerem o teste de normalidade Kolmogorov-Smirnov, método de correlação de Spearman, regressão linear, teste F e o teste t de Student.

A pesquisa foi desenvolvida em uma Escola Estadual, localizada na região central da cidade de Barra do Bugres, Mato Grosso, com 28 alunos da turma de $2^{\circ}$ ano do Ensino Médio, os quais responderam um questionário misto composto por 10 (dez) questões relacionadas ao tempo de estudo extraclasse dos conteúdos matemáticos ministrados em sala de aula.

As variáveis tempo de estudo extraclasse utilizando a internet e a não utilização da internet a respeito dos conteúdos matemáticos, foram extraídas por meio do questionário, e a variável nota bimestral na disciplina de Matemática foi disponibilizada pela professora da turma, obtendo assim, dados primários e secundários, respectivamente.

Após a coleta de dados, utilizamos o programa Minitab para verificar a normalidade dos dados pelo teste de Kol- 
mogorov-Smirnov com o nível de significância de 95\%, sendo esse o mais indicado para a quantidade de indivíduos da nossa população.

Posteriormente a essa análise, optamos em utilizar a Correlação de Spearman e a Regressão linear para as variáveis "tempo de estudo extraclasse utilizando a internet", "tempo de estudo extraclasse" e a "nota bimestral dos alunos" em relação aos conteúdos matemáticos e à disciplina de Matemática, respectivamente. Em seguida, utilizamos os testes t e o teste F para a variável Nota Bimestral dos alunos na disciplina de Matemática com o intuito de compararmos as médias das variáveis estudadas.

No caso em que os dados não formam um grupo "bem comportado" com alguns pontos muito afastado do restante, o coeficiente e de Spearman é o mais apropriado. O coeficiente o de Spearman mede a intensidade da relação entre variáveis ordinais e varia entre -1 e 1 . O sinal indica direção positiva ou negativa do relacionamento e o valor indica a força da relação entre as variáveis. Quanto mais próximo estiver desses extremos, maior será a relação entre as variáveis e quanto mais próximo de 0 menor será essa relação.

A correlação estatística tem como intuito medir a intensidade que mantêm unidas duas ou mais quantidades (variáveis), mesmo que isso não insinue em uma relação de causa-efeito. Podemos afirmar que existe correlação estatística entre duas variáveis ou mais quando uma delas está de alguma forma, relacionada com a outra.

Admitindo uma relação entre as variáveis x e y, as técnicas de Regressão podem ser usadas para encontrar um modelo, geralmente uma função matemática que expressa a dependência entre essas variáveis. Existem várias técnicas de regressão, porém, nessa pesquisa utilizamos a Regressão Linear expressada pela lei $y=a x+b$, em que a e $b$ são coeficientes angulares e lineares, respectivamente, a variável y é denominada variável resposta ou dependente e a variável $\mathrm{x}$ denominada variável explicativa ou independente.

O teste $t$ de Student tem como característica mais importante comparar as médias de duas amostras sejam elas independentes ou pareadas. Temos o teste $t$ unilateral e o bilateral, pois "se estivermos interessados em mostrar que um parâmetro é significativamente superior ou inferior a um determinado valor, teremos que realizar um teste unilateral e teremos uma única região de rejeição, do tamanho do nível de significância fixado" (Guimarães, 2008, p.146), porém, se quisermos verificar se há diferença entre um determinado parâmetro e um determinado valor (sem explicitar se é inferior ou superior), utilizamos o teste t bilateral. Assim, a "região de rejeição será dividida em duas partes iguais, nas extremidades da curva do teste, em que cada região de rejeição terá metade do nível de significância" (Guimarães, 2008, p. 146).

Para utilizarmos o teste $t$ para duas amostras, temos que inicialmente definir se utilizaremos o teste $t$ 'presumindo as variâncias equivalentes' ou 'presumindo variâncias diferentes'. Dessa forma, é necessário aplicar o teste F, o qual tem como característica principal comparar as variâncias das amostras, assim, após análise do teste F presumimos qual teste $t$ utilizar.

\section{Resultados e Discussão}

A disciplina de Matemática é tida por grande parte dos alunos como de difícil assimilação, sendo que uma das possibilidades para facilitar a compreensão dos conteúdos matemáticos é o estudo extraclasse. Assim, optamos em analisar o tempo de estudo extraclasse como fator de sucesso no processo de aprendizagem.

Com o objetivo de conhecer o grupo de alunos estudado, aplicamos um questionário e identificamos que os mesmos têm em média 16 anos de idade e todos residem na zona urbana de Barra do Bugres/MT. Desse grupo, 13 (treze) jovens são do sexo feminino e 15 (quinze) do sexo masculino. Dos 28 (vinte e oito) alunos pesquisados, 12 (doze) trabalham em média 3 (três) horas por dia e 16 (dezesseis) não trabalham. Em relação ao acesso à internet, 24 (vinte e quatro) acessamna, diariamente, e 4 (quatro) fazem o uso de maneira alternada, de forma que, desses 24 (vinte e quatro), 18 (dezoito) têm internet em suas casas.

Com os dados coletados, aplicamos o teste Kolmogorov-Smirnov com um nível de significância de 95\% e verificamos que os dados não seguem uma distribuição normal, pois identificamos o p-valor menor que 0,05 (nível de confiabilidade), assim, rejeitamos H0 (os dados seguem uma distribuição normal) e aceitamos H1 (os dados não seguem uma distribuição normal). Consideramos que a distribuição dos dados em estudo não provém de uma distribuição normal, então, utilizamos a correlação de Spearman.

De acordo com a Figura 1, é possível identificar a ocorrência da correlação entre a variável (i) 'tempo de estudo extraclasse utilizando a internet a respeito dos conteúdos matemáticos' e a variável (ii) 'nota bimestral dos alunos na disciplina de matemática', bem como ocorre uma correlação entre a variável (iii) 'tempo de estudo extraclasse dos conteúdos matemáticos' e a variável (ii) 'nota bimestral dos alunos na disciplina de matemática', levando em consideração o p-valor, temos todos os p-valores iguais à zero, menor que o nível de confiabilidade $(0,05)$. Desse modo, rejeitamos H0 (o coeficiente $\varrho$ é igual a zero) e aceitamos H1 (o coeficiente @ é diferente de zero), portanto, há uma relação de causalidade entre as variáveis aqui estudadas.

Por meio da correlação de Spearman verificamos que existe uma relação entre as variáveis, sendo assim, utilizamos a 


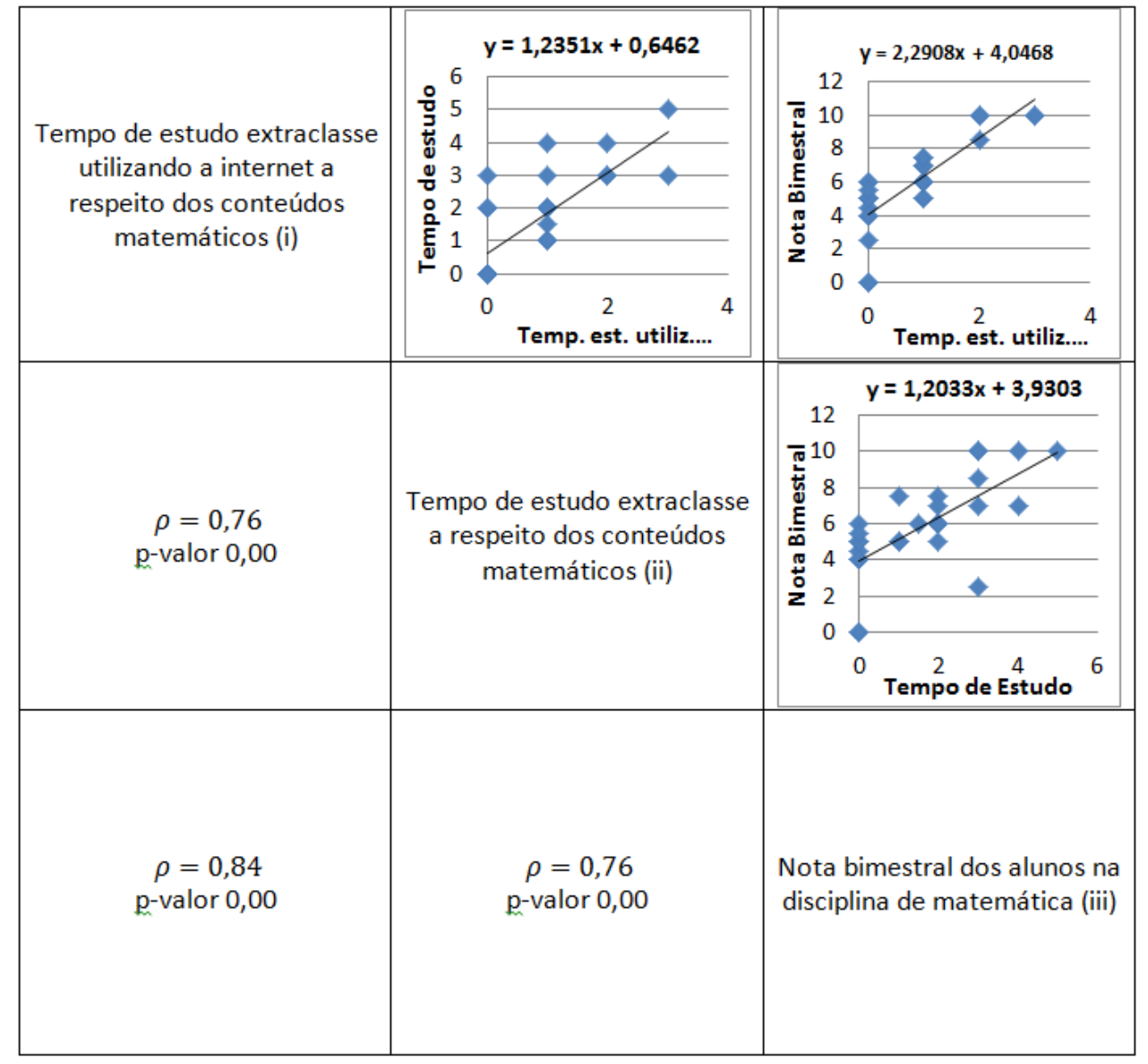

Figura 1 - Matriz de correlação e Regressão Linear Fonte: Próprio autor.

técnica de Regressão Linear para encontrar uma função matemática que expresse a lei de dependência entre as variáveis x e y.

A Figura 1 apresenta os gráficos de regressão das variáveis aqui estudadas, os quais descrevem aproximadamente uma função linear $y=a x+b$, em relação às variáveis explicativa ou independente $(x)$ e às variáveis resposta ou dependente (y). $O$ valor b é o coeficiente linear da reta, isto é, o valor onde a reta intercepta o eixo y e o valor a é o coeficiente angular da reta, pois é esse valor que define o ângulo entre a reta e o eixo $x$.

Analisando os diagramas de dispersão, verificamos que todos descrevem aproximadamente uma linha ascendente. Logo, é possível garantir que há relação entre as duas variáveis (iii) 'tempo de estudo extraclasse dos conteúdos matemáticos' e (ii) 'nota bimestral dos alunos na disciplina de matemática'. Identificamos que quanto mais tempo os alunos se dedicam ao estudo extraclasse, maior é a sua nota bimestral na disciplina de Matemática. Podemos afirmar que há relação entre as duas variáveis (i) 'tempo de estudo extraclasse utilizando a internet em relação aos conteúdos matemáticos' e a (ii) 'nota bimestral dos alunos na disciplina de Matemática' e que são diretamente proporcionais, ou seja, quanto mais tempo o aluno se dedica ao estudo extraclasse utilizando a internet, maior é a nota bimestral na disciplina de Matemática.

Nessa perspectiva, a utilização da internet amplia o espaço no processo de ensino-aprendizagem, pois o professor e os alunos podem se comunicar a qualquer momento, por meio de mensagens via e-mail, chats, redes sociais virtuais entre outros aplicativos, propiciando, assim, uma troca de saberes entre professor e aluno. Em consonância a isso, entendemos que a internet é uma das ferramentas tecnológicas que tem facilitado e motivado a interação e a promoção das relações interpessoais entre os envolvidos.

Os avanços tecnológicos, em particular os aparelhos eletrônicos como, os computadores, smartphones, tablet, e, também o advento da internet, propiciaram o desenvolvimento da sociedade, pois as informações que demoravam dias ou meses para chegar ao destino, agora, podem ser transmitidas quase que instantaneamente.

Com esses avanços, surgem as redes sociais virtuais, as quais oportunizam a comunicação e a troca de informação, vídeos, imagens e fotos a qualquer momento, bastando ter um dispositivo apropriado e ter acesso à internet, facilitando a comunicação e a informação entre elas.

A internet é "um ambiente social e acessível a todos os usuários, um espaço onde cada um determina e controla a informação de acordo com as suas necessidades e interesses" (SILVA e LUVIZOTTO, 2013, p. 1339), portanto, cada indivíduo 
escolhe o que, qual, onde e quando estudar, tornando-se assim, mais autônomo na construção do conhecimento.

De acordo com a Pesquisa Brasileira de Mídia (BRASIL, 2015), 37\% da população brasileira utiliza a internet diariamente. Entre os internautas, $92 \%$ estão conectados por meio de redes sociais, sendo as mais utilizadas o Facebook, o Whatsapp e o Youtube. Os jovens de 16 a 25 anos ficam conectados à internet em média 5h25min por dia, sejam nas redes sociais, jogos online, web sites, entre outros. Dessa forma, entendemos a importância da utilização da internet pelos professores e alunos na construção do conhecimento.

Por intermédio do questionário, verificamos que $82 \%$ dos alunos utilizam as redes sociais como Facebook e o Youtube para se comunicar e estudar conteúdos matemáticos. O Youtube tem se constituído uma rede social de compartilhamento de vídeos. O fato é que estas redes têm desempenhado um papel muito importante na Educação, pois nesses sites são compartilhados todos os tipos de vídeos, clipes de músicas, e vídeos que explicam conteúdos relacionados às várias áreas do conhecimento. Estes sites têm se convertido em poderosas ferramentas de propagação de informações.

Assim, as TDIC têm sido uma grande aliada do ensino e aprendizagem, pois de acordo com Almeida (2013),

[...] alunos e professores em contato com as TDIC tornam-se investigativos e não apenas receptivos, eles encontram novas fontes de ideias que vão além dos seus próprios pensamentos, começam a observar, refletir e atribuir significados, criando suas próprias conjecturas. Ou seja, a inserção das TDIC quebra paradigmas, podendo modificar qualitativamente a qualidade do ensino, tornando as aulas mais criativas, motivadoras e dinâmicas (ALMEIDA, 2013, p. 5).

A utilização das TDIC no ensino-aprendizagem tem propiciado um novo ambiente de ensino, pois o uso dos softwares e da internet por meio de sites, redes sociais, blogs, entre outros, tem facilitado a visualização, a comunicação, a criatividade e a motivação dos alunos na busca do conhecimento.

De acordo com os questionários, verificamos que $68 \%$ dos alunos são disciplinados a estudar conteúdos matemáticos mais de duas horas semanais fora da sala de aula, mostrando que a maioria dos analisados tem compromisso e consciência da importância do estudo extraclasse.

Com o intuito de verificarmos se há diferença das médias aritméticas das notas dos alunos na disciplina de Matemática, fizemos o teste $t$ de Student. Nesse caso, antes de fazermos o teste $t$, fizemos o teste $F$ para verificar qual teste $t$ teríamos de utilizar.

É importante ressaltar que esses dois testes só foram realizados para dados provenientes de uma distribuição normal. Nesse caso, fizemos o teste para a variável 'nota bimestral dos alunos na disciplina de Matemática', pois a mesma apresentou uma distribuição normal.

A variável 'nota bimestral dos alunos na disciplina de Matemática' foi dividida em duas variáveis, a 1ํㅗariável 'nota dos alunos que estudam conteúdos matemáticos menos de duas horas semanais fora da sala', e a $2^{\mathrm{a}}$ variável 'nota dos alunos que estudam conteúdos matemáticos mais de duas horas semanais fora da sala de aula'.

Ao realizarmos o teste $\mathrm{F}$ encontramos o p-valor igual a 0,504, maior que o nível de confiabilidade 0,05 , portanto, aceitamos H0 (as variâncias são equivalentes). Dessa forma, para essas variáveis, utilizamos o teste $t$ de Student 'para duas amostras presumindo as variâncias equivalentes'.

No teste citado, encontramos o p-valor igual a 0,002 menor que o nível de confiabilidade, portanto, rejeitamos H0 (não há diferença significativa entre as médias) e aceitamos H1 (há diferença significativa entre as médias). Nessa análise, podemos concluir, estatisticamente, que há diferença significativa nas médias das variáveis aqui estudadas.

Para verificarmos qual média é maior analisando o teste $t$ em relação às variâncias das variáveis, podemos afirmar que os alunos que estudam conteúdos matemáticos mais de duas horas semanais fora da sala de aula, tendem a ter uma nota bimestral maior na disciplina de Matemática, pois a média da $2^{\underline{a}}$ variável é maior que a da $1^{\underline{a}}$ variável.

Assim, podemos afirmar, estatisticamente, que as horas de estudos extraclasse semanais influenciam no rendimento escolar dos alunos (GONÇALVES et al., 2016), entendemos que essa estratégia é importante para a construção do conhecimento do estudante.

Num segundo momento, utilizamos a variável ‘nota bimestral dos alunos na disciplina de Matemática' e a dividimos em duas variáveis novamente, sendo a $1^{1}$ variável 'nota dos alunos que estudam conteúdos matemáticos utilizando a internet menos de duas horas semanais fora da sala de aula', e a $2^{\underline{a}}$ variável 'nota dos alunos que estudam conteúdos matemáticos utilizando a internet mais de duas horas semanais fora da sala de aula'. Com o intuito de compararmos as médias, utilizamos os testes $\mathrm{F}$ e t.

Com o teste $\mathrm{F}$ encontramos o p-valor igual a 0,02, menor que o nível de confiança, portanto rejeitamos H0 (as variâncias são equivalentes) e aceitamos H1 (as variáveis não são equivalentes). Concomitantemente, utilizamos o teste t para duas amostras presumindo variâncias diferentes. Com esse teste, encontramos o p-valor igual a 0,00, menor que o nível de confiança, portanto, rejeitamos H0 (não há diferença significativa entre as médias) e aceitamos H1 (há diferença significativa entre as médias).

Podemos concluir, estatisticamente, que há diferença significativa entre médias das notas dos alunos das duas variáveis. Os alunos que estudam conteúdos matemáticos utilizando a internet mais de duas horas semanais fora da sala de aula 
tendem a ter as notas bimestrais maiores em relação aos alunos que estudam conteúdos matemáticos utilizando a internet menos de duas horas semanais fora da sala de aula.

Logo, verificamos que o tempo de estudo extraclasse tem contribuído para a construção do conhecimento dos alunos aqui pesquisados, e a internet tem motivado o estudo extraclasse, contribuindo assim, para a construção do seu conhecimento.

\section{Considerações finais}

Com o objetivo de analisar o tempo de estudo extraclasse como fator de sucesso no processo de aprendizagem na disciplina de Matemática dos alunos do $2^{\circ}$ ano do Ensino Médio de uma Escola Estadual da cidade de Barra do Bugres, Mato Grosso, pudemos observar que as variáveis (i) 'tempo de estudo extraclasse utilizando a internet a respeito dos conteúdos matemáticos', (ii) 'nota bimestral dos alunos na disciplina de Matemática' e (iii) 'tempo de estudo extraclasse em relação aos conteúdos matemáticos', apresentam correlação. Verificamos que existe uma dependência das variáveis y em relação às variáveis $x$ e que quanto mais a variável $x$ cresce, mais a variável y também cresce. Sendo assim, podemos considerar que, estatisticamente, o tempo de estudo extraclasse foi um fator de sucesso para a melhoria no desempenho dos alunos, e que contribuiu nas notas bimestrais na disciplina de Matemática.

Após as análises dos testes t de Student, a correlação de Spearman e a regressão linear, acreditamos que seja importante o estudo extraclasse a respeito dos conteúdos matemáticos. Verificamos que os alunos que estudaram esses conteúdos mais de duas horas semanais fora da sala de aula, bem como os que se utilizaram das TDIC no estudo extraclasse, apresentaram as maiores notas nessa disciplina.

Sendo assim, é importante a utilização das TDIC nas práticas pedagógicas, pois essas permitem que os alunos busquem opções de aprendizado bem como a oportunidade de fazer o feedback a qualquer momento que sentir necessidade.

É importante que os professores se qualifiquem no que concerne à utilização das TDIC, para que possam proporcionar, um novo ambiente escolar, o qual será mais atrativo e motivacional, tanto para os alunos como para os próprios professores.

\section{Referências}

ALMEIDA, H. R. F. L. O Uso das Tecnologias Digitais da Informação e Comunicação na Aula de Cálculo 1 a Distância. In: XVII Encontro Brasileiro de Estudantes de Pós-Graduação em Educação Matemática, 2013, Vitória - ES. Anais do XVII EBRAPEM, 2013.

BRASIL, Instituto Nacional de Estudos e Pesquisas Educacionais Anísio Teixeira. Programa Internacional de Avaliação de Alunos PISA. 2012. Disponível em <http://portal.inep.gov.br/internacional-novo-pisa-resultados>. Acesso em: 15 abr. de 2016.

BRASIL. Secretaria de Comunicação Social. Pesquisa Brasileira de Mídia - PBM. 2015. Disponível em: <http://www.secom.gov.br/ atuacao/pesquisa/lista-de-pesquisas-quantitativas-e-qualitativas-de-contratos-atuais/pesquisa-brasileira-de-midia-pbm-2015.pdf/ view>. Acesso em: 13 mai. de 2016.

GONÇALVES, M. P. G.; SIPAÚBA, J. S.; QUEIROZ JR., J. V.; MOUSINHO, J. M. R. P.; AGUIAR FILHO, U. N. Influência do Tempo de Estudo no Rendimento do Aluno Universitário. Revista Fundamentos, v. 2, p. 1, 2016.

GUIMARÃES, P. R. B. Métodos Quantitativos Estatísticos. 1. ed. Curitiba: IESDE Brasil S.A., 2008, 252 p.

POCINHO, R. F. S; GASPAR, J. P. O uso das TIC e as alterações no espaço educativo. Exedra Journal, n. 6, p. 143-154, 2012.

SILVA, D. R.; LUVIZOTTO, Caroline Kraus. Uso de Redes Sociais da Internet Como Ferramentas de Apoio na Educação: Estudos e Possibilidades. Colloquium Humanarum, v. 10, p. 1333-1341, 2013.w 